\title{
Localización de sitios en mapas raster para minimizar impactos de flujo
}

\author{
Pablo Vanegas $^{1}$, Dirk Cattrysse ${ }^{2}$, Jos Van Orshoven ${ }^{3}$ \\ ${ }^{1}$ Facultad de Ingeniería, Universidad de Cuenca, Av. 12 de Abril s/n, Cdla. Universitaria, Cuenca, \\ Ecuador. \\ ${ }^{2}$ Centre for Industrial Management, Katholieke Universiteit Leuven, Celestijnenlaan 300A, 3001 \\ Heverlee (Leuven), Belgium \\ ${ }^{3}$ Department of Earth and Environmental Sciences, Katholieke Universiteit Leuven, Celestijnenlaan \\ 200E, 3001 Heverlee (Leuven), Belgium
}

E-mail: pablo.vanegas@ucuenca.edu.ec

Fecha de recepción: 16 de septiembre 2010 - Fecha de aceptación: 13 de octubre 2010

\section{RESUMEN}

La conservación del medio ambiente y la planificación del uso de la tierra con frecuencia requieren identificar sitios que contribuyan a reducir al mínimo el flujo de sedimentos en los ríos. Las iniciativas de forestación pueden considerar la reducción de sedimentos entre sus objetivos. Este artículo propone una formulación de Programación Entera (IP) y un método heurístico para determinar áreas a ser reforestadas con la finalidad de minimizar la carga de sedimentos a la salida de una cuenca hidrográfica. Aunque la estructura de ambos métodos se puede aplicar para modelar otros tipos de flujo, éstos se enfocan en la minimización de la descarga de sedimentos. Los métodos propuestos hacen uso de un mapa raster con la dirección de flujo y que cubre la cuenca hidrográfica. Este mapa permite construir una estructura de datos en árbol de tal forma que la celda a la salida de la cuenca corresponde con el nodo raíz de este árbol. Los resultados obtenidos con la formulación entera y con el método heurístico concuerdan con evaluaciones de expertos que consideran los niveles de erosión, la pendiente y las distancias a los lechos de los ríos. Estos resultados permiten concluir que ambos métodos son apropiados para la minimización del flujo de sedimentos. Por otro lado, la heurística requiere mucho menos tiempo de cómputo, razón por la cual este método de solución es el más adecuado para aplicaciones a nivel regional.

Palabras clave: Optimización, localización de sitios, minimización de flujo, programación entera, método heurístico, forestación.

\begin{abstract}
Policy and decision makers dealing with environmental conservation and land use planning often require identifying potential sites for contributing to minimize sediment flow reaching riverbeds. This is the case of reforestation initiatives, which can have sediment flow minimization among their objectives. This paper proposes an Integer Programming (IP) formulation and a Heuristic solution method for selecting a predefined number of locations to be reforested in order to minimize sediment load at a given outlet in a watershed. Although the core structure of both methods can be applied for different sorts of flow, the formulations are targeted to minimization of sediment delivery. The proposed approaches make use of a Single Flow Direction (SFD) raster map covering the watershed in order to construct a tree structure so that the outlet cell corresponds to the root node in the tree. The results obtained with both approaches are in agreement with expert assessments of erosion levels, slopes and distances to the riverbeds, which in turn allow concluding that the studied approaches are suitable for minimizing sediment flow.
\end{abstract}

Keywords: Optimization, site location, flow minimization, integer programming, heuristic method, forestation. 


\section{INTRODUCCIÓN}

De acuerdo a la revisión de literatura, hasta la presente fecha no se han desarrollado métodos que explícitamente consideren la interacción entre celdas como parte del proceso de ubicación o selección de áreas. Sin embargo existen aplicaciones que no se limitan a considerar la información intrínseca de cada celda, sino también la Interacción Espacial (IE) entre celdas. De esta forma la interacción produce un impacto fuera de las áreas seleccionadas. En este tipo de problemas la inclusión de una celda como parte del área objetivo modifica también las condiciones de una o más celdas vecinas o incluso alejadas. Este comportamiento se ilustra con el ejemplo donde la forestación tiene como objetivo reducir la carga de sedimentos a la salida de una cuenca. Bajo una representación raster, cuando una celda es seleccionada para ser reforestada, las condiciones de transporte y retención de sedimentos van a cambiar. Esto implica que una mayor o menor cantidad de sedimento será entregada a las celdas vecinas, por lo tanto las condiciones de estas últimas también serán modificadas. El presente trabajo busca que los métodos propuestos estén en capacidad de responder a la pregunta: ¿Dónde están ubicadas las mejores celdas a ser reforestadas de tal forma que el flujo de sedimentos se reduce a un mínimo a la salida de una cuenca hidrográfica?

Varios modelos de simulación han sido desarrollados durante los últimos años con el propósito de representar interacción entre celdas. Aplicaciones de Autómatas Celulares (AC) han recibido particular atención en literatura para áreas relacionadas con la modelación de flujo, por ejemplo dinámica de canales (Murray y Paola, 1994), evolución de paisaje (Coulthard y col., 1999), hidrodinámica (Parsons y Fonstad, 2007), flujos de lava (Di-Gregorio y Serra, 1999), erosión del suelo (D'Ambrosio y col., 2001), carga de sedimentos (Coulthard y col., 2002), entre otros. De todas formas no se han desarrollado métodos que explícitamente consideren la selección de sitios óptimos en ambientes donde existe IE.

El presente artículo tiene como objetivo adaptar y aplicar conceptos de AC para desarrollar un método heurístico que permita minimizar el impacto externo producido por un área seleccionada. La aplicación está enfocada en localizar las mejores celdas a ser reforestadas, es decir, celdas donde se modifiquen las condiciones de producción y transporte de sedimentos de tal forma que el flujo sea reducido a un mínimo a la salida de una cuenca hidrográfica. Dado que el flujo no es de naturaleza lineal, se aplica una función convexa lineal por segmentos para determinar la entrega de flujo desde una celda hacia sus vecinos. La función convexa lineal por segmentos necesita dos puntos de corte para definir tres segmentos, a su vez, cada segmento se asocia a un factor específico de transporte. Los puntos de corte, los factores asociados a cada segmento y la producción de flujo cambian en las celdas reforestadas. Dado que estos cambios generan impactos fuera de sitio debido a la interacción espacial, es necesario hacer uso de un enfoque que permita modelar este comportamiento dinámico. A pesar de que la heurística propuesta no es un Autómata Celular en sí mismo, se siguen aplicando conceptos de estado de celdas, de vecindad y de interacción.

Los resultados obtenidos con un método de programación entera son utilizados como referencia para medir la exactitud del enfoque heurístico. La formulación entera se modela como un problema general de redes de flujo donde se satisfacen las restricciones de capacidad en los enlaces y de balance de masa en todas las celdas. La siguiente sección resume trabajos pertinentes desarrollados anteriormente en materia de arrastre de flujo. Mientras que la primera parte de la sección 3 detalla los datos y su representación, la segunda, tercera y cuarto parte explican los enfoques propuestos para localizar celdas óptimas a ser reforestadas. La sección 4 muestra los resultados obtenidos con la formulación entera (IP) y con la heurística basada en Autómatas Celulares (CAMF). Por último, la sección 5 expone las conclusiones.

\section{REVISIÓN DE LITERATURA}

Tomando en cuenta que Relación Espacial (RE) especifica cómo los objetos se encuentran en el espacio en relación a otros objetos de referencia, el concepto de Interacción Espacial (IE) designa la 
existencia de relaciones causales en el espacio o la existencia de procesos de difusión espacial. El análisis espacial considera las transformaciones, manipulaciones y métodos que se pueden aplicar a los datos geográficos para agregar valor a ellos, para apoyar las decisiones y para revelar patrones y anomalías que no son inmediatamente obvias (Longley y col., 2005). Modelación, una tema importante relacionado con el análisis espacial, se define (Van Orshoven, 2007) como la descripción de fenómenos del mundo real en términos abstractos, matemáticos o gráficos con el fin de promover su comprensión. Debido a la diversidad de modelos existentes, una clasificación que diferencia los modelos estáticos de los modelos de simulación es útil. Cuando los problemas del mundo real no se pueden representar, por ejemplo con modelos analíticos, la simulación es la única alternativa para apoyar la toma de decisiones. La simulación se puede definir como una técnica que imita el funcionamiento de un sistema del mundo real a medida que evoluciona en el tiempo. Un modelo de simulación por lo general toma la forma de un conjunto de supuestos acerca de la operación del sistema, expresado como relaciones matemáticas o lógicas entre los objetos de interés (Winston, 1994). Hasta ahora, las interacciones producidas por el arrastre de sedimentos no se han representado con métodos analíticos. En este sentido, la formulación IP propuesta en este artículo considera la interacción entre celdas con el propósito de reducir al mínimo la carga de sedimentos (impacto fuera del área) en la salida de una cuenca.

\subsection{Modelo de dirección única de flujo}

Una característica común está presente en las aplicaciones de AC para la simulación y predicción de flujo: la dirección de flujo es una variable en las reglas de decisión. Sin embargo, existen varios métodos que permiten obtener mapas con la dirección del flujo calculada en base a información de relieve. La forma tradicional para determinar la dirección de flujo es la ruta descendiente con mayor pendiente, llamada la Dirección única de flujo (Single Flow Direction -SFD-). Sin embargo, los defectos de los algoritmos de SFD han sido discutidos por varios autores (Bogaart y col., 2006; Qin y col., 2007; y Failfield y Leymarie, 1991). Uno de los estudios muestra que los algoritmos SFD pueden ser apropiados para las regiones convergentes del paisaje, pero no lo son para laderas divergentes (Bogaart y col., 2006). Desmet y Govers (1996) realizan una comparación entre 6 algoritmos de dirección de flujo basados en modelos de elevación digital (Digital Elevation Models -DEM-). Estos algoritmos son aplicados para identificar los lugares susceptibles a la formación de cárcavas. Las predicciones requieren de información de pendientes así como del área de contribución para cada celda. Los resultados muestran que los modelos SFD predicen erosión por cárcavas en lugares poco probables y los autores afirman que los modelos SFD son sensibles a errores en topografía. Los modelos de Dirección de flujo múltiple (Multiple Flow Direction -MFD-) son capaces de identificar en forma más precisa las zonas susceptibles de erosión por cárcavas, a pesar de que la cárcava en sí mismo no es ubicada con precisión. Por otro lado, los modelos SFD permiten identificar concavidades más cerca de la realidad. En la misma línea de Desmet y Govers (1996), cuyo trabajo se centra en la identificación de las características físicas, Wolock y McCabe (1995) concluyen que la eficiencia del modelo TOPMODEL y la simulación de rutas de flujo se vieron afectados ligeramente cuando el índice topográfico se calculó con un algoritmo SFD en lugar de un algoritmo MFD. Las diferencias en la eficiencia del modelo y en simulación de rutas de flujo calculadas con SFD and MDF esencialmente desaparecen cuando el modelo es calibrado mediante el ajuste de parámetros hidráulicos sub-superficiales.

La ausencia de métodos que consideren optimización espacial combinada con la reducción del flujo de sedimentos se explica por la complejidad de los modelos de programación entera (IP) para la identificación de áreas para la reducción de flujo y por los altos requerimientos de memoria. La aplicación de algoritmos MFD combinado con las demandas de optimización espacial, requeriría de una cantidad extremadamente elevada de recursos computacionales. Dado que según Wolock y McCabe (1995) los algoritmos SFD siguen siendo válidos para la simulación de las trayectorias de flujo, y teniendo en cuenta los requerimientos computacionales de los algoritmos MFD, los métodos propuestos en este artículo emplean direcciones de flujo calculadas de antemano con algoritmos SFD. 


\subsection{Problema de la red de flujo}

Una red de flujo considera [Ahuja y col., 1993] una red dirigida $G=(N, A)$ definida por un conjunto $N$ de $n$ nodos y un conjunto $A$ de $m$ arcos. Cada arco $(i, j) \in A$ tiene asociado un costo por unidad de flujo $c_{i}$, una capacidad máxima $u_{i j}$ y una cantidad mínima $l_{i j}$ que debe fluir en el arco. Una cantidad $b(i)$ se asocia a cada nodo $i$; si $b(i)>0, i$ es un nodo de abastecimiento, si $b(i)<0, i$ es un nodo de demanda, y si $b(i)=0, i$ es un nodo de transbordo.

Si bien el costo de flujo entre los nodos puede variar de forma lineal con la cantidad de flujo, funcionales no lineales o funciones lineales por segmentos también se aplican en situaciones reales. En un modelo convexo lineal por segmentos (Ahuja y col., 1993) el costo de cada arco $C_{i j}\left(x_{i j}\right)$ se descompone en máximo $p$ segmentos lineales: $\left(0=d^{0}{ }_{i j}\right)<\left(d^{l}{ }_{i j}\right)<\left(d_{i j}^{2}\right)<\ldots$ denotan los puntos de corte de la función convexa, donde el costo varía de forma lineal en el intervalo $\left[d^{k-1} i, d_{i j}^{k}\right.$. Por lo tanto, para especificar una función de costo lineal en segmentos, es necesario especificar los puntos de corte y la pendiente de cada segmento lineal entre dos puntos sucesivos de corte. En este sentido, un flujo $x_{i j}$ puede ser descompuesto en varios segmentos, donde cada uno representa el flujo entre dos puntos de corte $d^{k-1}{ }_{i j}, d^{k}{ }_{i j}$. Si $y_{i j}^{k}$ denota el flujo en el segmento $k$, es decir, entre $d^{k-1}{ }_{i j} y d_{i j}^{k}$, el flujo en cada segmento se determina de la siguiente manera:

$$
y_{i j}^{k}=\left\{\begin{array}{rlr}
0 & \text { if } \quad x_{i j} \leq d_{i j}^{k} \\
x_{i j}-d_{i j}^{k} & \text { if } \quad d_{i j}^{k-1} \leq x_{i j}<d_{i j}^{k} \\
d_{i j}^{k}-d_{i j}^{k-1} & \text { if } \quad x_{i j} \geq d_{i j}^{k}
\end{array}\right.
$$

Tomando en cuenta que $C_{i j}$ es una función de costo convexa, entonces $C_{i j}\left(x_{i j}\right)=\sum_{k=1}^{p} c_{i j}^{k} *^{k}{ }_{i j}$. Por lo tanto, la formulación de las ecuaciones 1, 2, 3 transforma el problema de flujo de costo convexo a un problema de flujo de costos mínimos. La ecuación 2 representa restricciones de balance de masa; el primer término corresponde al flujo total de salida y el segundo al flujo de entrada al nodo $i$. Por último, la ecuación 3 controla la cantidad que se arrastrará desde el nodo $i$ al $j$ a través del segmento $k$.

$$
\begin{gathered}
\sum_{\text {Minimizar: }} \sum_{\{j:(i, j) \in A\}}^{p} c_{k=1}^{k} y_{i, j}^{k} \\
\text { s.t: }\{j:(i, j) \in A\} k=1 \\
0 \leq y_{i, j}^{k} \leq d_{i j}^{k}-d_{i j}^{k}-\sum_{\{j:(j, i) \in A\}} \sum_{k=1}^{p} y_{j, i}^{k}=b(i) \quad \forall(i, j) \in A \quad k=1, \ldots, p
\end{gathered}
$$

\section{MATERIALES Y MÉTODOS}

\subsection{Descripción de los datos}

Los métodos propuestos necesitan diez mapas raster (matrices) para identificar celdas idóneas a ser forestadas con la finalidad de minimizar la carga de sedimentos a la salida de una cuenca: 1) dirección del flujo; 2) producción de flujo $\left.\left(\alpha_{j}\right) ; 3\right)$ factor de flujo $\left.\left(\gamma_{j, k}\right) ; 4\right)$ punto de corte \# $\left.1\left(\sigma_{j, 1}\right) ; 5\right)$ punto de corte \# $\left.2\left(\sigma_{i, 2}\right) ; 6\right)$ producción modificada de flujo $\left.\left(\beta_{i}\right) ; 7\right)$ factor modificado de flujo $\left.\left(\delta_{i, k}\right) ; 8\right)$ punto modificado de corte \# $1\left(\sigma_{i, 3}\right)$; 9) punto modificado de corte \# $2\left(\sigma_{i, 4}\right)$; y 10$)$ lechos de río. En consideración a que los estados de las celdas se modifican cuando éstas son reforestadas (seleccionadas como parte de la solución óptima) con el objetivo de reducir al mínimo la carga de sedimentos, los primeros 5 mapas reflejan el estado original en un año 0 y los siguientes 4 mapas representan un estado modificado (reforestación) en un año $n$ posterior a la reforestación. Los símbolos griegos que se utilizan en la formulación matemática se asocian con el mapa correspondiente. A continuación se describe cada mapa. 
1. La dirección del flujo (Flow Direction -FD-) se calcula en base a mapas de elevación. FD utiliza una dirección única de flujo en cada celda (SFD), calculada de acuerdo a las direcciones de flujo descendientes presentes en la ventana de 3-por-3 alrededor de la celda actual (Vecindad de Moore). El método SFD asigna una dirección de flujo único, es decir hacia la celda con mayor diferencia en elevación dentro del vecindario.

2. Producción de flujo $\left(\alpha_{j}\right)$ es un término general que se asocia con los niveles de flujo de cualquier clase, producidos localmente en la celda $j$. En el problema en curso se refiere a los sedimentos o erosión producidos en la celda.

3. Factor de flujo $\left(\gamma_{i, k}\right)$ cuantifica la fracción de sedimento arrastrado desde la celda $j$ a su vecino $k$ de menor altitud. En los problemas donde el relieve es importante, como en el problema que nos ocupa, la pendiente en la celda puede actuar como un factor de entrega de flujo.

4. Punto de corte \#1 $\left(\sigma_{j, 1}\right)$ es el primer punto de corte de una función convexa lineal por segmentos la cual modela la naturaleza no lineal del flujo de sedimentos en la celda $j$.Los puntos de corte son necesarios para definir los segmentos lineales en las funciones de arrastre de sedimento. En el presente artículo $\sigma_{j, 1}$ determina la capacidad de retención: si el flujo total en la celda $j$ es menor que $\sigma_{j, 1}$, no existe flujo hacia las celdas vecinas.

5. Punto de corte \# $2\left(\sigma_{i, 1}\right)$ es el segundo punto de corte de la función que determina el flujo de sedimentos desde la celda $j$.

6. Producción modificada de flujo $\left(\beta_{i}\right)$, factor modificado de flujo $\left(\delta_{i, k}\right)$, punto modificado de corte \# $1\left(\sigma_{i, 3}\right)$, y punto modificado de corte \# $2\left(\sigma_{i, 4}\right)$ son los nuevos valores para la producción de flujo, factor de flujo, punto de corte \# 1 y punto de corte \# 2 cuando la celda $j$ es seleccionada para ser reforestadas. Por lo tanto estos valores corresponden a nuevos parámetros de la función convexa lineal por segmentos.

7. Este mapa está representado por una cuadrícula binaria en la que las celdas con valor 1 representan cauces de los ríos.

\subsection{Representación del problema}

La identificación de las mejores celdas a ser forestadas necesita en primera instancia especificar una celda de salida donde converge el flujo total. Tomando en cuenta que el mapa con la dirección única de flujo (SFD) asigna una ruta única desde cada celda hacia la celda vecina más baja, es factible representar este mapa con una estructura en árbol en la cual el nodo raíz corresponde a la celda de salida. La Figura 1a esquematiza los cinco mapas iniciales utilizados para minimizar el flujo de sedimentos. La estructura de árbol subyacente en la Figura 1b se construye a partir del mapa con dirección única de flujo. Cada nodo del árbol corresponde a una celda, por lo tanto los valores asociados a las celdas en cada uno de los 10 mapas raster se referencian desde el nodo correspondiente; por ejemplo, los nodos del árbol en la Figura $1 \mathrm{~b}$ se asocian con el factor de flujo entre el nodo $i$ y el nodo $j\left(\gamma_{i, j}\right)$ y la producción de flujo en el nodo i $\left(\alpha_{i}\right)$.

\subsection{Función convexa lineal por segmentos}

La formulación matemática y el método heurístico propuestos hacen uso de una función convexa lineal por segmentos $(g)$ para modelar el flujo de sedimento entre nodos. Esta función descompone el flujo continuo en un número finito de segmentos convexos, tal que $g$ es lineal en cada segmento. La Figura 2a muestra el flujo de entrada y salida del nodo $j$ y la Figura $2 \mathrm{~b}$ ilustra la función compuesta por dos puntos de corte $\left(\sigma_{i, 1}, \sigma_{i, 2}\right)$ y tres segmentos $\left(y_{i, k, 1}, y_{j, k, 2}, y_{i, k, 5}\right)$ que modela el flujo entre los nodos $j, k$. El flujo de entrada es definido como la Acumulación Efectiva en el nodo $j\left(A E_{j}\right)$, la cual corresponde a la suma del flujo proveniente de los nodos hijos ( $h, i$ en 2a), más el flujo producido en el propio nodo $(j)$. 


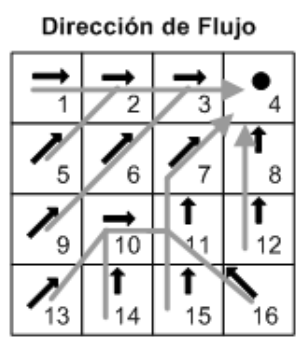

Producción de Flujo
\begin{tabular}{|r|r|r|r|}
\hline 1.0 & 1.0 & 1.0 & 1.0 \\
1 & 2 & 3 & 4 \\
\hline 1.0 & 1.0 & 1.0 & 1.0 \\
5 & 6 & 7 & 8 \\
\hline 1.0 & 1.0 & 1.0 & 1.0 \\
9 & 10 & 11 & 12 \\
\hline 1.0 & 1.0 & 1.0 & 1.0 \\
13 & 14 & 15 & 16 \\
\hline
\end{tabular}

Factor de flujo
\begin{tabular}{|r|r|r|r|}
\hline 1.0 & 0.5 & 0.5 & 1.0 \\
1 & 2 & 3 & 4 \\
\hline 1.0 & 0.4 & 0.7 & 0.2 \\
5 & 6 & 7 & 8 \\
\hline 1.0 & 0.2 & 0.7 & 0.2 \\
9 & 10 & 11 & 12 \\
\hline 1.0 & 1.0 & 1.0 & 1.0 \\
13 & 14 & 15 & 16 \\
\hline
\end{tabular}

Punto de corte 1
\begin{tabular}{|r|r|r|r|}
\hline 0.5 & 0.5 & 0.5 & 0.5 \\
1 & 2 & 3 & 4 \\
\hline 0.5 & 0.5 & 0.5 & 0.5 \\
5 & 6 & 7 & 8 \\
\hline 0.5 & 0.5 & 0.5 & 0.5 \\
9 & 10 & 11 & 12 \\
\hline 0.5 & 0.5 & 0.5 & 0.5 \\
13 & 14 & 15 & 16 \\
\hline
\end{tabular}

Punto de corte 2

\begin{tabular}{|r|r|r|r|}
\hline 1.0 & 1.0 & 1.0 & 1.0 \\
1 & 2 & 3 & 4 \\
\hline 1.0 & 1.0 & 1.0 & 1.0 \\
5 & 6 & 7 & 8 \\
\hline 1.0 & 1.0 & 1.0 & 1.0 \\
9 & 10 & 11 & 12 \\
\hline 1.0 & 1.0 & 1.0 & 1.0 \\
13 & 14 & 15 & 16 \\
\hline
\end{tabular}

b) Representación en árbol del mapa de dirección de flujo

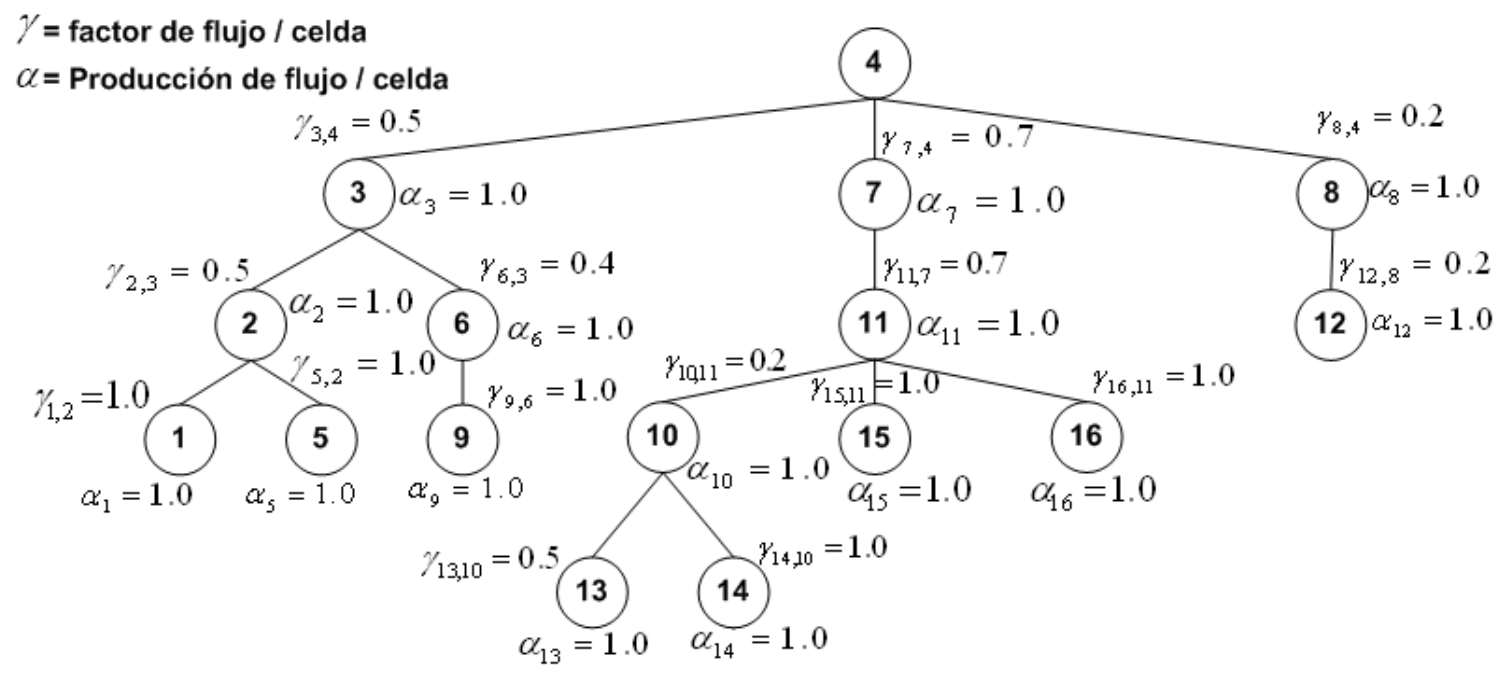

Figura 1. a) Datos y b) Representación del problema.

El flujo de salida del nodo $j$ y entregado al nodo padre $k$ depende de $\left.A E_{j}: 1\right)$ Cuando $A E_{j}<\sigma_{j, 1}$, el flujo en el segmento $1\left(y_{j, k, 1}\right)$ es igual a $A E_{j}$; el flujo en los segmentos $y_{i, k, 2}$ y $y_{j, k, 5}$ es igual a 0.2$)$. Si $\sigma_{j, 1}$ $=<A E_{j}<\sigma_{j, 2}$ el flujo a lo largo de los tres segmentos se distribuye de la siguiente manera: $y_{j, k, 1}=\sigma_{j, 1}$; $\left.y_{j, k, 2}=A E_{j}-\sigma_{j, 1} ; y_{j, k, 5}=0.3\right) \mathrm{Si} A E_{j} \geq \sigma_{j, 2}$, el flujo en el segmento 1 es igual a $\sigma_{j, 1}$, el flujo en el segmento 2 es igual a $\sigma_{j, 2}-\sigma_{j, 1}$ y por el segmento 3 fluye una cantidad igual a $A E_{j}-\sigma_{j, 2}$. La Figura $2 \mathrm{c}$ especifíca el flujo por los tres segmentos en función de la acumulación efectiva $\left(\mathrm{AE}_{j}\right)$ en el nodo $j$.

Por lo tanto, una fracción $\gamma_{j, k}$ del flujo en el segmento 2 más todo el flujo en el segmento 5 es entregado al nodo padre $k$, es decir: $g_{k}=\gamma_{j, k} * y_{j, k, 2}+y_{j, k, 5}$. Esto implica que el sedimento que excede $\sigma_{i, 2}$ y arrastrados a través del segmento $y_{i, k, 5}$ fluye en su totalidad al nodo padre. Por otro lado el sedimento en el segmento $1\left(y_{i, k, 1}\right)$ es retenido. Estas características se presentan en la Figura $2 \mathrm{~b}$, la que acumula el flujo a lo largo de los distintos segmentos para finalmente determinar la cantidad de flujo que se entregará al nodo padre $k$. La Figura $2 \mathrm{~b}$ representa la capacidad de retención $\left(\sigma_{i, 1}\right)$, así como el flujo que excede el punto de corte $2\left(\sigma_{j, 2}\right)$. Los segmentos $y_{j, k, 2}$ o $y_{j, k, 5}$ podrían no existir (igual a 0 ) cuando el flujo no supera a $\sigma_{j, l}$ o $\sigma_{j, 2}$ respectivamente.

\subsection{Formulación matemática de la red de flujo}

La formulación entera para la minimización del flujo de sedimentos hace uso de una función convexa lineal por segmentos, donde cada enlace entre un par de nodos $j, k$ en la representación original en árbol (Figura 1) se sustituye por un conjunto de cinco enlaces: $y_{j, k, 1}, y_{j, k, 2}, y_{j, k, 3}, y_{j, k, 4}, y_{j, k, 5}$ (Figura 3) para la entrega de flujo desde el nodo $j$ al nodo $k$. Mientras por una parte los segmentos $y_{j, k, 1}, y_{j, k, 2}$ son utilizados para entregar el flujo de sedimentos al nodo $k$ cuando el nodo $j$ no se reforesta, los segmentos $y_{j, k, 3}, y_{j, k, 4}$ se utilizan cuando el nodo $j$ es reforestado. El segmento $y_{j, k, 5}$ recoge el flujo que excede el punto de corte $\sigma_{j, 2}$ cuando $j$ no está reforestado o $\sigma_{j, 4}$ cuando $j$ se ha seleccionado como uno 
de los mejores nodos a ser reforestados. Esto permite modelar la función convexa lineal por segmentos como parte de la formulación entera.

a)

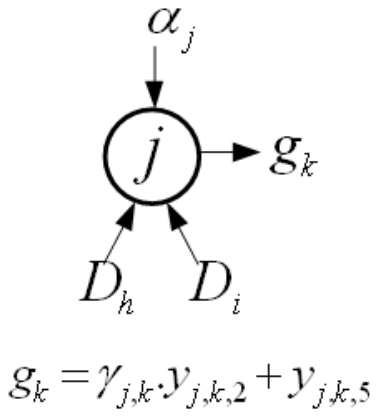

b)

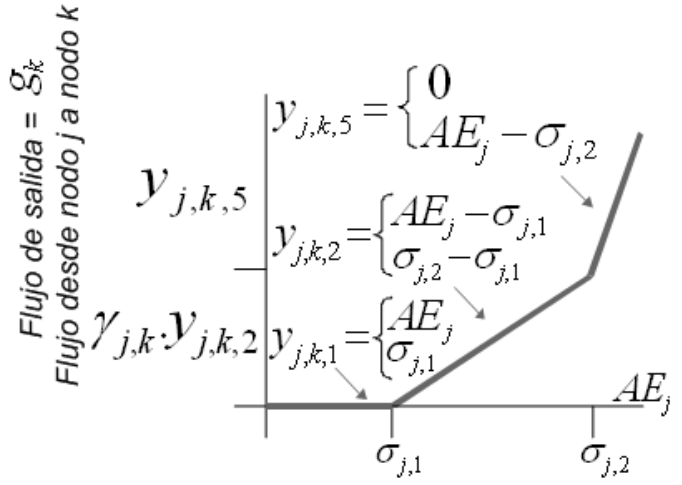

Acum. Ef. $\left(A E_{j}\right)=D_{h}+D_{i}+\alpha_{j}$

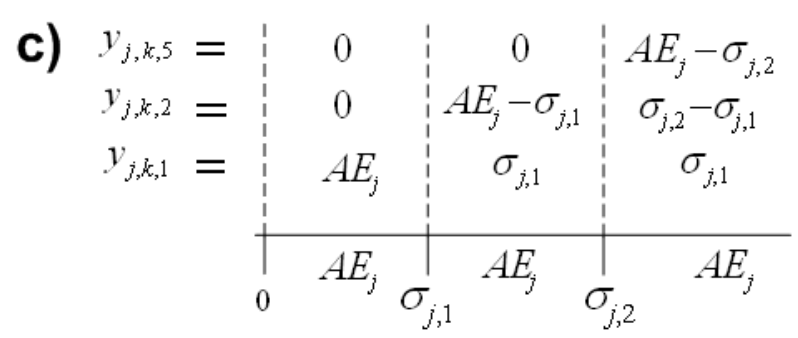

$$
\begin{aligned}
& A E_{j}=D_{h}+D_{i}+\alpha_{j}
\end{aligned}
$$

Figura 2. Función convexa lineal por segmentos para modelar el flujo de sedimentos de una celda a otra.

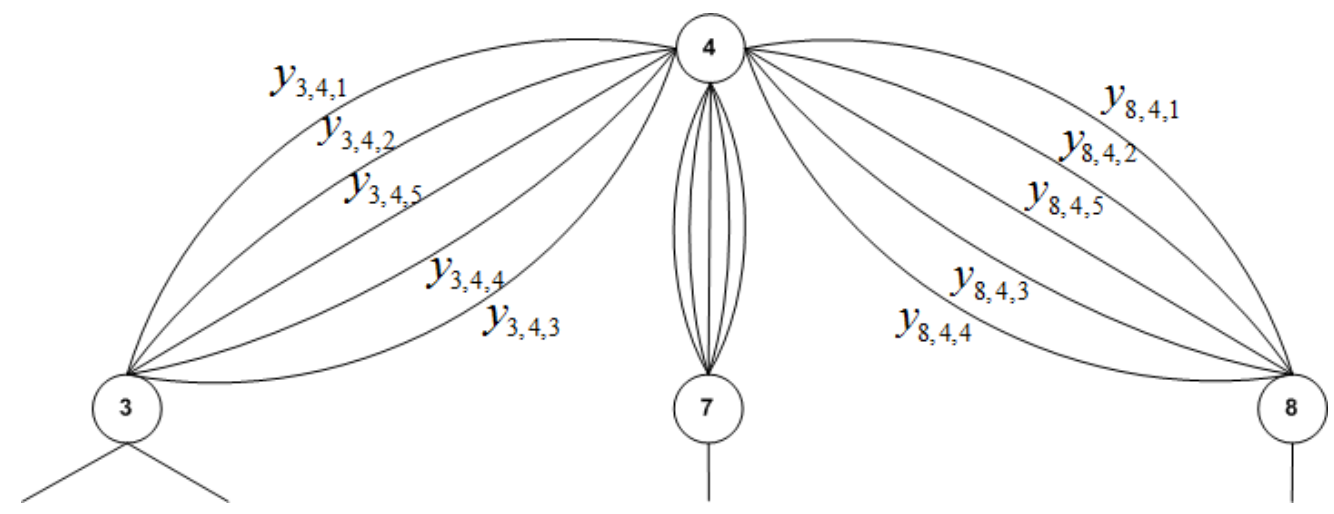

Figura 3. Segmentos para modelar flujo entre dos nodos.

La siguiente notación facilita la exposición del modelo matemático basado en la formulación general de la red de flujo con funciones convexas lineales por segmentos.

$\alpha_{j} \quad$ Producción de flujo en el nodo $j$

$\beta_{j} \quad$ Producción de flujo en el nodo j luego de ser reforestado 
$\gamma_{j, k} \quad$ Factor de transporte de flujo desde el nodo $\mathrm{j}$ al $\mathrm{k}$

$\delta_{j, k} \quad$ Factor de transporte de flujo desde el nodo $\mathrm{j}$ al $\mathrm{k}$, luego de reforestar el nodo $\mathrm{j}$

$\sigma_{j, 1} \quad$ Primer punto de corte de la función convexa lineal por segmentos en el nodo j

$\sigma_{j, 2}$ Segundo punto de corte de la función convexa lineal por segmentos en el nodo j

$\sigma_{j, 3}$ Primer punto de corte de la función convexa lineal por segmentos en el nodo j cuando este nodo es parte de la solución (reforestado)

$\sigma_{j, 4} \quad$ Segundo punto de corte de la función convexa lineal por segmentos en el nodo $\mathrm{j}$ cuando este nodo es parte de la solución (reforestado)

$f_{j} \quad$ Variable binaria que indica si el nodo j es parte de la solución

$y_{j, k, 1} \quad$ Flujo desde el nodo $\mathrm{j}$ al nodo $\mathrm{k}$ a través del segmento 1 (s1) en la función convexa lineal por segmentos, donde s1 está en el intervalo $\left[0 . \sigma_{\mathrm{j}, 1}\right]$

$y_{j, k, 2} \quad$ Flujo desde el nodo j al nodo k a través del segmento 2 (s2) en la función convexa lineal por segmentos, donde s2 está en el intervalo $\left[\sigma_{\mathrm{j}, 1} \cdot \sigma_{\mathrm{j}, 2}\right]$

$y_{j, k, 3}$ Flujo desde el nodo $\mathrm{j}$ al nodo $\mathrm{k}$ a través del segmento 3 (s3) en la función convexa lineal por segmentos, donde $\mathrm{s} 3$ está en el intervalo $\left[0 . \sigma_{\mathrm{j}, 3}\right]$

$y_{j, k, 4} \quad$ Flujo desde el nodo $\mathrm{j}$ al nodo $\mathrm{k}$ a través del segmento 4 (s4) en la función convexa lineal por segmentos, donde s4 está en el intervalo $\left[\sigma_{\mathrm{j}, 3} . \sigma_{\mathrm{j}, 4}\right]$

$y_{j, k, 5}$ Flujo desde el nodo $\mathrm{j}$ al nodo $\mathrm{k}$ a través del segmento 5 (s5) en la función convexa lineal por segmentos. El flujo que excede $\sigma_{\mathrm{j}, 2} \mathrm{O} \sigma_{\mathrm{j}, 4}$ será transportado por este segmento

$r_{j} \quad$ Valor binario que indica si el nodo j está disponible para ser reforestado. Por ejemplo nodos que no pertenecen a cauces de ríos

$\varphi \quad$ Número de celdas objetivo

La Figura 4a esquematiza una función convexa lineal por segmentos para nodos no reforestados y la Figura $4 \mathrm{~b}$ la función para nodos reforestados. El eje de las abscisas en las Figuras 4a y 4b representa el flujo de sedimentos de entrada al nodo $j$, el cual corresponde al flujo proveniente de los nodos hijos más el flujo producido en el mismo nodo $j\left(\alpha_{j} o \beta_{j}\right)$. Cuando el nodo $j$ no está reforestado (Figura 4a), el flujo entregado a su padre (nodo $k$ ) sigue el procedimiento explicado en la sección 2.3. Cuando el nodo $j$ se encuentra reforestado, la cantidad de flujo de salida (Figura $4 \mathrm{~b}$ ) se determina mediante una función similar a la de la sección 2.3, pero en esta ocasión con parámetros modificados. La modificación de los parámetros en la función convexa lineal para nodos reforestados se realiza de la siguiente forma: 1) Los puntos de corte: $\sigma_{j, 1}$ y $\sigma_{j, 2}$ se sustituyen por $\sigma_{j, 3}$ y $\sigma_{j, 4}$ respectivamente; 2) Los segmentos: $y_{j, k, 1}$ y $y_{j, k, 2}$ se sustituyen por $y_{j, k, 2}$ y $\left.y_{j, k, 4} ; 3\right)$ Los factores de flujo: $\gamma_{j, k}$ por $\left.\delta_{j, k} ; 4\right)$ La producción de flujo: $\alpha_{j}$ por $\beta_{j}$. Por lo tanto, cuando el flujo se arrastra a lo largo de los segmentos $y_{j, k, 2}$ (no reforestados) o $y_{i, k, 4}$ (reforestados), estos segmentos son respectivamente multiplicados por los factores $\gamma_{i, k}$ y $\delta_{j, k}$. Como estos factores tienen valores entre 0 y 1 , el flujo puede ser parcialmente transportado. Por el contrario, el flujo a lo largo del segmento $y_{j, k, 5}$ siempre se transporta en su totalidad desde el nodo $j$ al nodo $k$ (Figuras $4 \mathrm{a}$ y $4 \mathrm{~b}$ ). Por último, el flujo a lo largo de $y_{j, k, 1}$ (nodos no reforestados, Figura 4a) o $y_{j, k, 3}$ (nodos reforestados, Figura 4b) es retenido en el nodo $j$.

La función objetivo (ecuación 4) de la formulación entera (IP) reduce al mínimo la acumulación efectiva (AE) de flujo en el nodo raíz. Las variables $f_{j}$ tienen un valor 1 si el nodo $j$ es seleccionado como parte de la solución (reforestado), o un valor 0 si no está seleccionado (no forestado). Mientras que el flujo de entrada al nodo raíz proveniente de sus hijos $j$ (primera parte de la ecuación 4), se calcula multiplicando el flujo en los segmentos $y_{j, r a i z, 2}$ y $y_{j, r a i z, 4}$ por sus respectivos factores de flujo 
$\left(\gamma_{j, \text { raiz }}, \delta_{j, \text { raiz }}\right)$, la cantidad producida en el nodo raíz se especifica por $\beta_{\text {raíz }}$ cuando el nodo raíz es forestado $\left(r_{a i z}=1\right)$, o por $\alpha_{\text {raiz }}$ cuando el nodo raíz no está reforestado $\left(1-f_{\text {raíz }}=1\right)$.
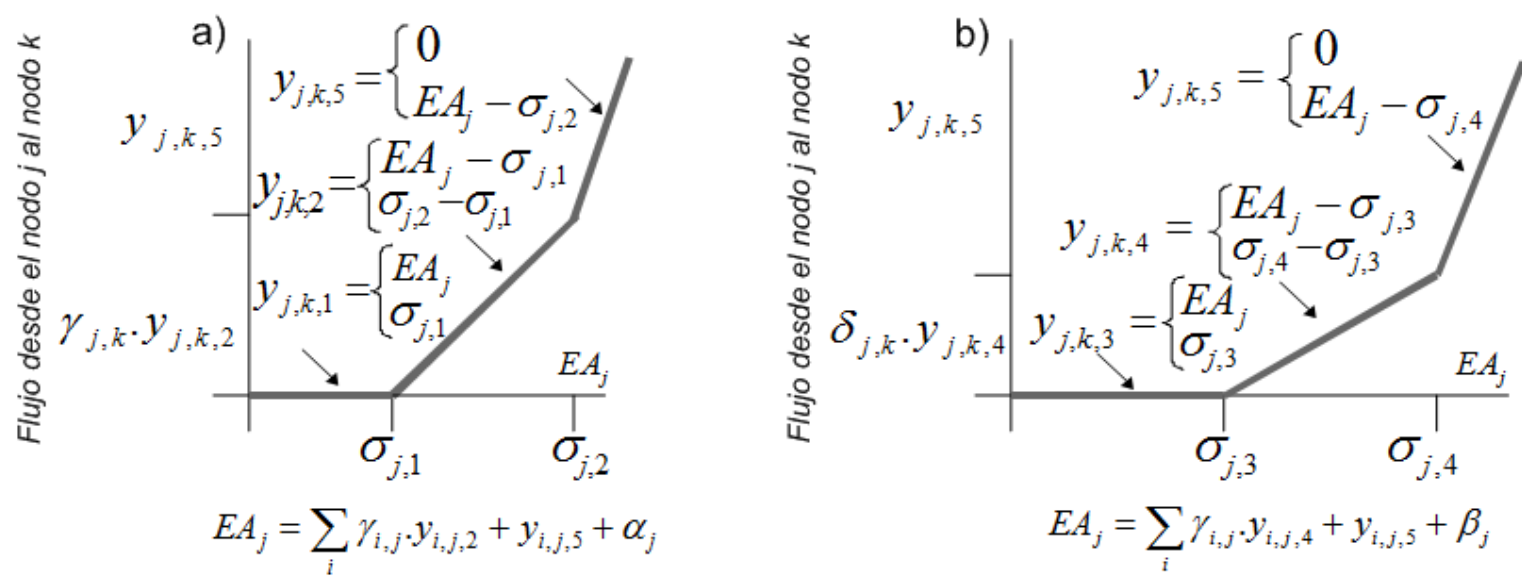

Figura 4. a) Función convexa lineal por segmentos para nodos no reforestados. b) Función convexa lineal por segmentos para nodos reforestados.

Las ecuaciones 5 y 6 equilibran los flujos de entrada y salida en el nodo $j$. En la ecuación 5, la entrada es igual al flujo entregado por sus nodos hijos más la producción de flujo (erosión) en el nodo $j$. Las ecuaciones 5 y 6 representan la acumulación efectiva (AE). Estas ecuaciones consideran que un nodo puede ser reforestado $\left(f_{i}=1 ; \delta_{i, j}\right)$ o no $\left(1-f_{i}=1 ; \gamma_{i, j}\right)$. Aunque los segmentos $y_{i, i, 1}$ y $y_{i, i, 3}$ también pueden mantener valores mayores que 0 , no se consideran en la ecuación 5 de tal manera que el flujo en el nodo $i$ se retiene cuando es menor que $\sigma_{i, 1}$ o $\sigma_{i, 3}$ (véase la Figura 4).

La ecuación 6 calcula el flujo de sedimentos desde el nodo $\mathrm{j}$ al nodo $\mathrm{k}$, donde $\mathrm{k}$ es el nodo padre. A pesar de que en esta ecuación todos los segmentos se suman, solamente $y_{j, k, 1}, y_{j, k, 2}$ y $y_{j, k, 5}$ se utilizan cuando el nodo $j$ no está reforestado mientras los segmentos $y_{j, k, 3}$ y $y_{j, k, 4}$ tienen un valor 0 (no se utilizan). Por el contrario, la formulación hace uso de segmentos $y_{j, k, 3}, y_{i, k, 4}$ y $y_{i, k, 5}$ cuando el nodo $j$ es seleccionado para ser reforestado. El segmento $y_{i, j, 5}$ mantiene un valor mayor que 0 solamente cuando el flujo de salida excede $\sigma_{i, 2}$ o $\sigma_{i, 4}$.

Minimizar:

$\sum_{j}\left(\gamma_{j, \text { raiz }} * y_{j, \text { raiz }, 2}+\delta_{j, \text { raiz }} * y_{j, \text { raiz }, 4}+y_{j, \text { raiz }, 5}\right)+\alpha_{\text {raiz }} *\left(1-f_{\text {raiz }}\right)+\beta_{\text {raiz }} * f_{\text {raiz }}$

s.t:

$$
\begin{array}{ll}
E A_{j}=\sum_{i}\left(\gamma_{i, j} * y_{i, j, 2}+y_{i, j, 5}+\delta_{i, j} * y_{j, r a i z, 4}\right)+\alpha_{j} *\left(1-f_{j}\right)+\beta_{j}^{*} f_{j} & \forall_{j} \\
E A_{j}=y_{j, k, 1}+y_{j, k, 2}+y_{j, k, 3}+y_{j, k, 4}+y_{j, k, 5} & \forall_{j, k} \\
y_{j, k, 1} \leq \sigma_{j, 1} *\left(1-f_{j}\right) & \forall_{j, k} \\
y_{j, k, 2} \leq\left(\sigma_{j, 2}-\sigma_{j, 1}\right) *\left(1-f_{j}\right) & \forall_{j, k} \\
y_{j, k, 3} \leq \sigma_{j, 3} * f_{j} & \forall_{j, k} \\
y_{j, k, 4} \leq\left(\sigma_{j, 4}-\sigma_{j, 3}\right) * f_{j} & \forall_{j, k} \\
r_{j}+f_{j} \leq 1 & \forall_{j} \\
f_{j} \in\{0,1\} & \forall_{j}
\end{array}
$$




$$
\sum_{j=1}^{\kappa} f_{j}=\varphi
$$

La cantidad de flujo a lo largo de cada segmento se determina por las ecuaciones 7 y 8 si el nodo $j$ no es reforestado $\left(1-f_{j}=1\right)$. Considerando que las restricciones en la ecuación 7 garantizan que el flujo de sedimentos a lo largo de $y_{j, k, 1}$ debe ser menor o igual que $\sigma_{i, 1}$; las restricciones en la ecuación 8 garantizan que el flujo en $y_{j, k, 2}$ debe estar entre $\sigma_{i, 1}$ y $\sigma_{i, 2}$. La misma explicación es válida para las ecuaciones 9 y 10 en el caso de que el nodo $j$ es forestado $\left(f_{j}=1\right)$.

El conjunto de variables en la ecuación 11 define la disponibilidad de los nodos a ser reforestados con la finalidad de reducir al mínimo la carga de sedimentos en el nodo raíz, es decir, si $r_{j}$ $=1$ el nodo $j$ no está disponible (por ejemplo nodos en lechos ríos) para ser forestado, y cuando $\mathrm{r}=0$ está disponible. Por último, la ecuación 12 define las variables $f$ como binarias, y la ecuación 13 limita el número de nodos seleccionados a $\varphi$. En la ecuación 13, $\kappa$ es el número total de nodos disponibles.

\subsection{Heurística basada en Autómatas Celulares para minimización de flujo (CAMF)}

Adicional a la representación que se explica en la sección 3.2, el método heurístico propuesto requiere una lista de nodos ordenados (L-lev) de acuerdo a sus niveles en la estructura en árbol. El esquema en la Figura 5 muestra esta lista, donde cada posición apunta a su correspondiente nodo en el árbol.

El método CAMF se divide en dos procesos principales: 1) cálculo de la Acumulación Efectiva (AE) inicial de flujo en cada nodo dentro de la representación en árbol; y 2) localización de los mejores nodos a ser reforestadas para minimizar el flujo que llega a la raíz (salida). La heurística hace uso de la lista L-lev con el fin de calcular la $\mathrm{AE}$ en cada nodo en el árbol.

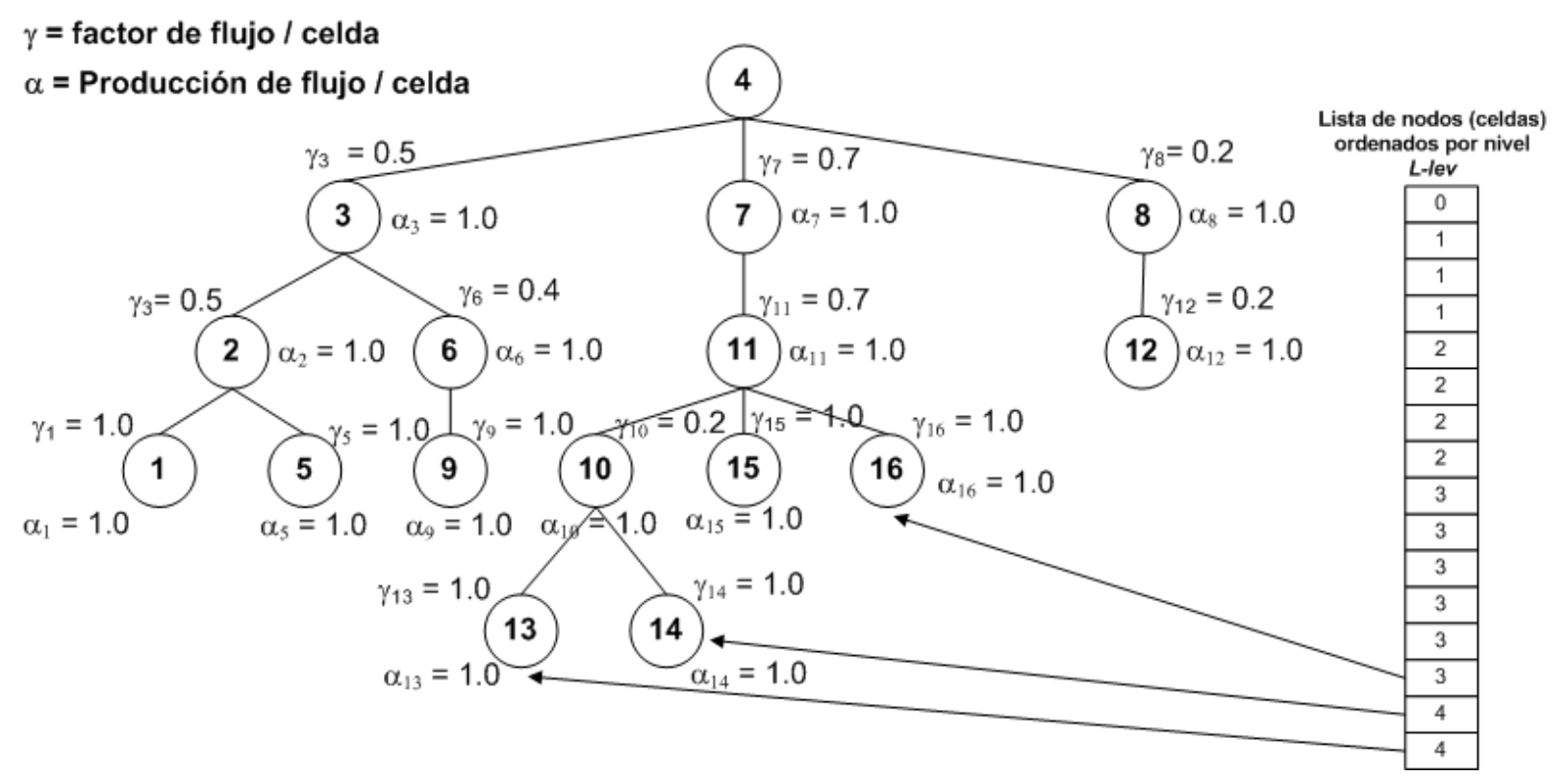

Figura 5. Lista de nodos.

\subsubsection{Cálculo de la Acumulación Inicial Efectiva (AE)}

De acuerdo con la representación en la Figura 1, la acumulación efectiva (AE) de sedimento en los nodos sin hijos (hojas) es igual al flujo producido en estos nodos sin tomar en cuenta cualquier otra contribución o interacción. La AE para los nodos que no son hojas se calcula nivel por nivel, de abajo hacia arriba, teniendo en cuenta la producción de flujo (erosión) en el nodo $j\left(\alpha_{j}\right)$ y el flujo proveniente desde sus hijos. En la función convexa lineal por segmentos en la Figura 2b, el flujo de sedimentos de entrada (eje X) es igual a la AE en el nodo $j$. El cálculo de la AE se realiza nivel por nivel, desde las hojas hasta alcanzar el nodo raíz. Luego de este proceso, el flujo acumulado en forma efectiva se pueda calcular según la ecuación 14, donde el estado actual en el nodo $j\left(Q^{t+1}\right)$ es función del caudal 
producido en el nodo en una iteración anterior $\left(Q_{i}^{t}=\alpha_{j}\right)$ y el flujo proveniente de los nodos hijos en la iteración actual $\left(\Omega^{t+1}\right)$. De esta manera, la ecuación 14 tiene en cuenta la influencia del estado de los nodos hijos en la iteración actual $(\mathrm{t}+1)$. La Figura 6 muestra los pasos seguidos para calcular la $\mathrm{AE}$ inicial en los nodos de los dos primeros niveles del árbol en la Figura 1. Un proceso similar es necesario para calcular la $\mathrm{AE}$ hasta alcanzar la raíz. Este procedimiento hace uso de una función convexa lineal por segmentos que guarda el estado original (no reforestado) en todos los nodos, a fin de determinar el flujo de salida. El cálculo de la AE inicial (año 0) está implementada en el algoritmo 3 , donde el mapa inicial de producción de sedimentos $(\alpha j)$ se toma como la $\mathrm{AE}$ de partida $\left(A E_{j}=\alpha_{j}\right.$ $\forall j)$.

$$
Q_{j}^{t+1}=f\left(Q_{j}^{t}, \Omega_{j}^{t+1}\right)
$$
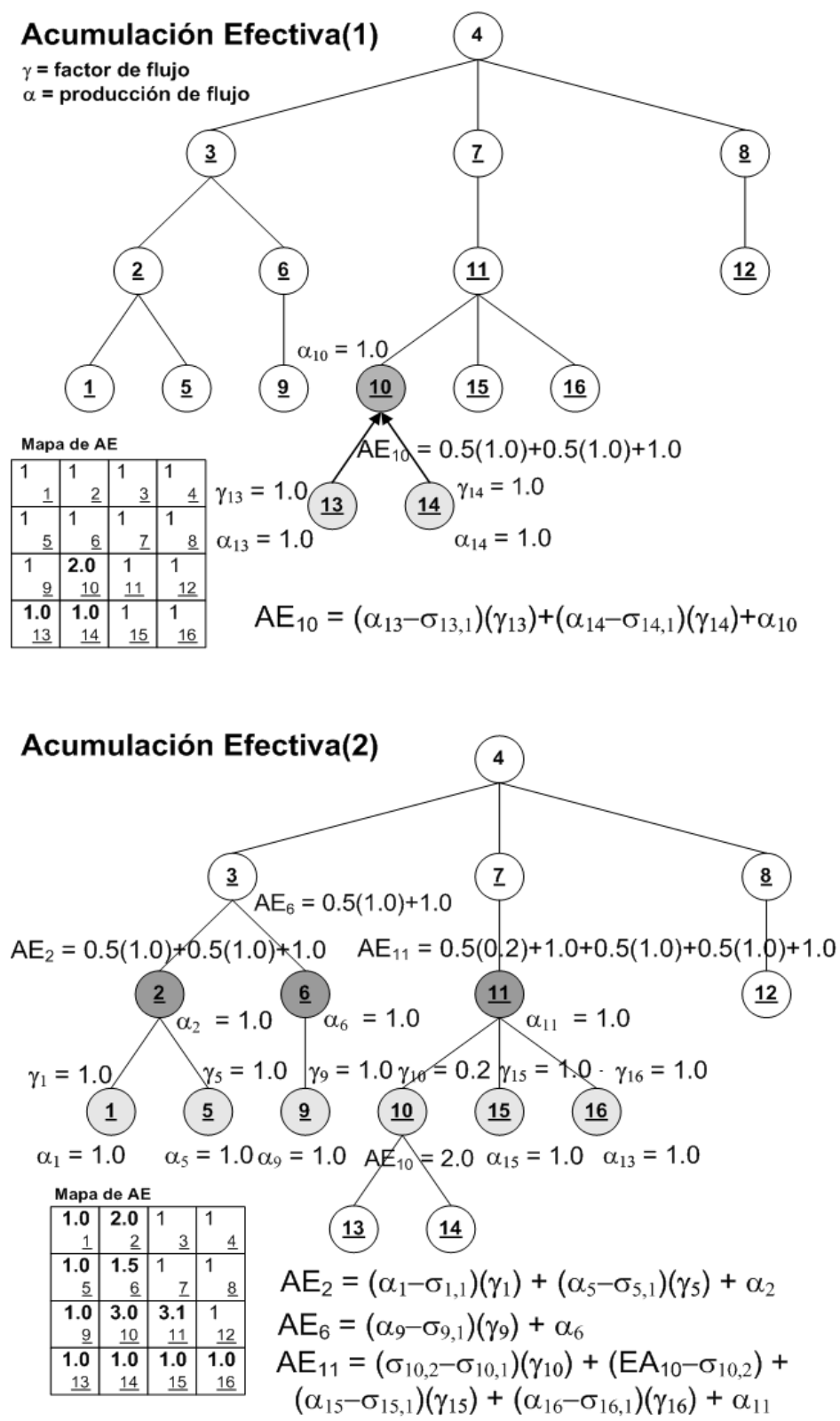

Figura 6. Ejemplo de cálculo de la Acumulación Efectiva (AE).

3.5.2. Localización de los nodos para minimizar flujo 
Los procedimientos necesarios para determinar los mejores nodos a ser reforestados se explican en la primera parte de esta sección. La segunda parte detalla cómo estos procedimientos operan en conjunto con el fin de localizar los mejores nodos para reducir al mínimo la carga de sedimentos en el nodo raíz (salida).

\section{Diferencia en el nodo raíz}

Cuando un nodo $j$ es reforestado se produce una diferencia en la Acumulación Efectiva (EA) de flujo en el nodo raíz. El cálculo de esta diferencia requiere, en primer lugar, determinar la cantidad de flujo que sale del nodo $j$ bajo dos estados: 1) estado inicial (anteriorD en el algoritmo 2) en el año 0: cuando el nodo $j$ conserva los parámetros iniciales de de la función convexa lineal $\left(\sigma_{i, 1}, \sigma_{i, 2}\right)$, el factor $\left(\gamma_{i, k}\right)$ y la producción de flujo $\left(\alpha_{j}\right)$; y 2) estado modificado (nuevoD en el algoritmo 2 ): cuando $\sigma_{i, 1}$ y $\sigma_{i, 2}$ se sustituyen respectivamente por $\sigma_{i, 3}$ y $\sigma_{i, 4}$.

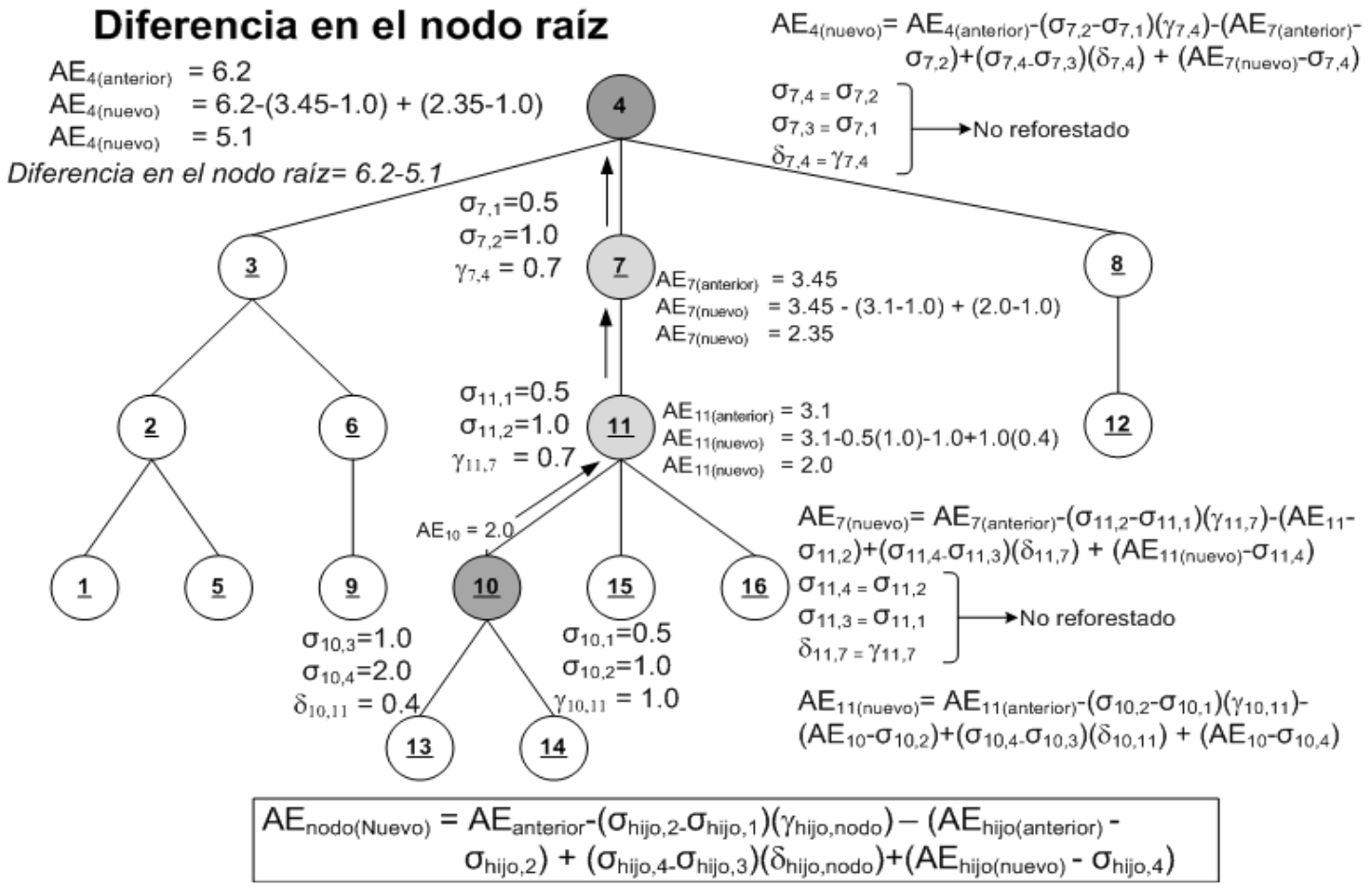

Figura 7. Cálculo de la diferencia de flujo en el nodo raíz.

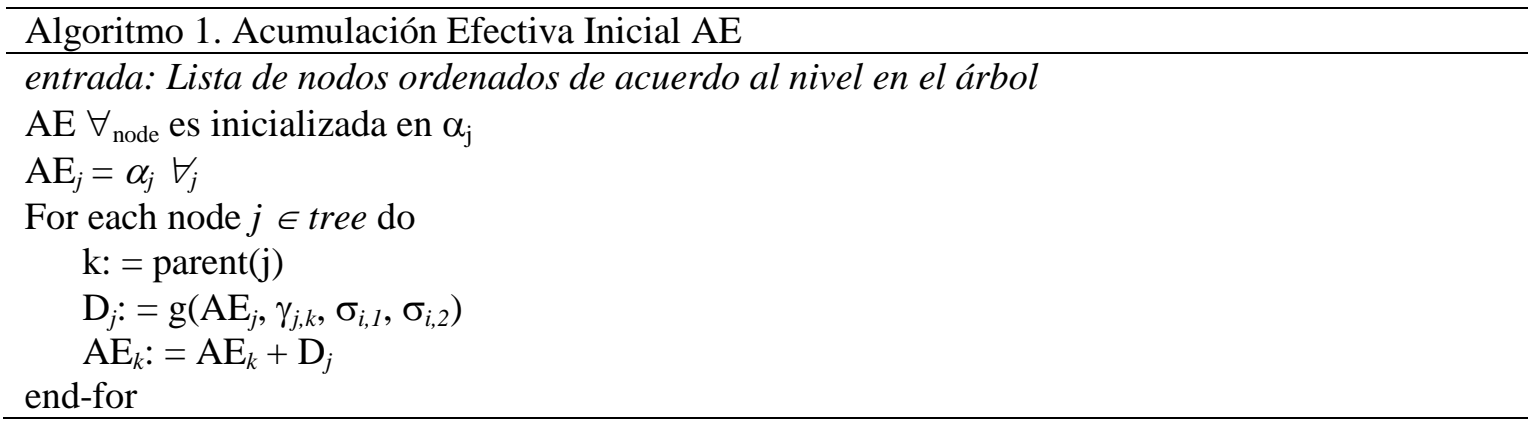

La producción de flujo $\alpha_{j}$ se sustituye por $\beta_{i}$ en la $\mathrm{AE}$ del nodo $j\left(\mathrm{~A} E_{j}-\alpha_{j}+\beta_{j}\right.$ en el algoritmo 2$)$. Una vez que la diferencia de flujo es determinada para el nodo seleccionado $j$ (nuevaAEj), esta diferencia se resta de la $\mathrm{AE}$ en el nodo padre $\left(\mathrm{AE}_{k}=\mathrm{AE}_{k}\right.$ - anteriorD + nuevoD). La actualización del nodo padre considera su estado actual: reforestado $(j \in S)$ o no $(j \notin S)$. El proceso descrito se repite para cada nodo del árbol, proceso que sigue el sendero desde el nodo seleccionado $j$ hasta el nodo raíz, de tal manera que la diferencia de flujo en la raíz es calculada (difFlujo ${ }_{k}$ en el algoritmo 2). El conjunto $\mathrm{S}$ 
se mantendrá vacío cuando las diferencias en el nodo raíz se calculan por primera vez. En las siguientes iteraciones el conjunto $\mathrm{S}$ almancenará los nodos seleccionados para ser reforestados.

En la Figura 7, el nodo \# 10 es seleccionado para ser reforestado, y desde allí se utilizan los nodos en el sendero (\# 11, \# 7) hasta llegar a la raíz (\# 4) para calcular la diferencia de flujo en la salida. Para facilitar la explicación, la Figura 7 asume dos condiciones extremas: 1) El flujo desde un nodo es siempre superior al punto de corte \# $2\left(\sigma_{\text {node, } 2}\right)$ o al punto de corte \# $4\left(\sigma_{\text {node, },}\right)$, y 2$)$ los nodos a lo largo del sendero (\# 11, \# 7) no están reforestados. Sin embargo, se pueden considerar condiciones distintas (por ejemplo, reforestación en los nodos en el sendero) al cambiar las fórmulas en la Figura 7 de acuerdo a la función convexa lineal por segmentos para la entrega de flujo (Figura 4). Si el nodo \# 10 es reforestado, la nueva AE en el padre (nodo \# 11) se determina de acuerdo a la función convexa lineal por segmentos en la Figura 4a. Mientras el cálculo de la AE en el nodo \# 11 considera que el estado del nodo \# 10 ha cambiado debido a la reforestación, el cálculo de la $\mathrm{AE}$ en el nodo \# 7 considera el estado inicial (no reforestado) en el nodo \# 11; del mismo modo, la AE en el nodo \# 4 se calcula manteniendo el estado inicial en el \# 7.

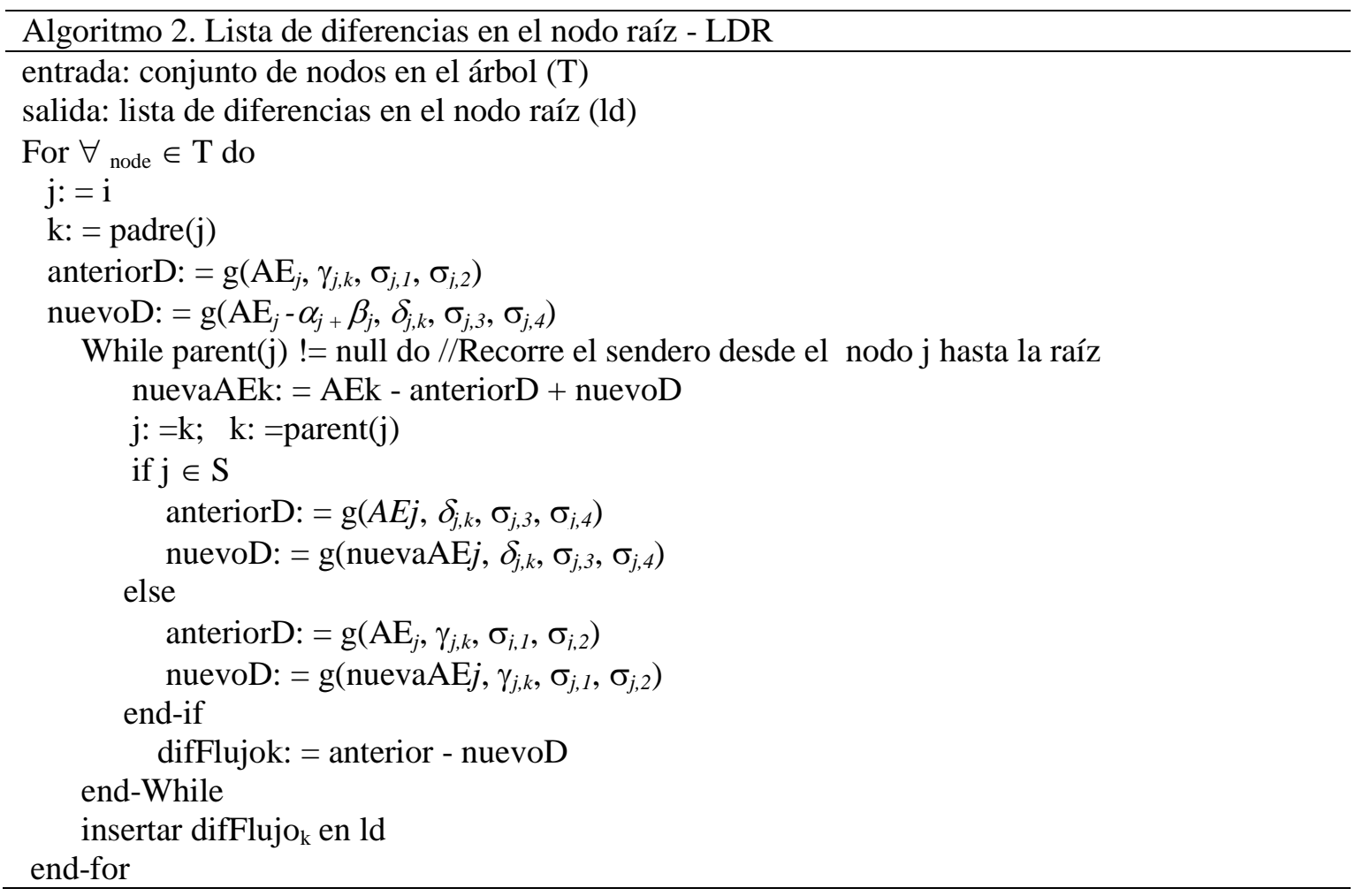

\section{Localización de los mejores nodos}

De acuerdo a los procedimentos explicados, cuando un nodo particular es reforestado, su nueva función convexa lineal por segmentos producirá una diferencia en el flujo de sedimentos en el nodo raíz (LDR, algoritmo 2). Esta diferencia se calcula para cada nodo en el árbol y se almacena en una lista ordenada (ld en el algoritmo 2). En una primera iteración, el cálculo de las diferencias considera que solamente el nodo seleccionado es reforestado y el resto de nodos no sufren cambios, es decir $\mathrm{S}=\varnothing$ en la primera llamada del algoritmo 2 (LDR) en el algoritmo 3 (LBN).

El procedimiento para la localización de los mejores nodos (LBN) empieza por calcular la lista de diferencias en el flujo que ingresa al nodo raíz (ld) bajo la suposición inicial de que $S=\varnothing$ en el algoritmo 2. Tomando en cuenta que los nodos en la parte superior de ld son los que corresponden con las mayores diferencias de flujo de sedimentos en el nodo raíz, estos nodos son candidatos a ser parte de la solución final. Dado que es probable que más de un nodo se pueda situar en la parte superior de la lista, mejoresNodos marca el punto inicial de los nodos con las mayores diferencias (Figura 8). Si dentro de los nodos ubicados entre la parte superior y mejoresNodos (conjunto B en el algoritmo 3) 
existe una relación padre - hijo, los padres son removidos de $\mathrm{B}$ ( $\mathrm{B}=\mathrm{B}$ - ([padres de $\left.\left.\forall_{\text {nodo }} \in \mathrm{B}\right] \in \mathrm{B}\right)$ en el algoritmo 3). Un nodo en el nivel más bajo es preferido debido a que la modificación de su producción de erosión y los parámetros de entrega de flujo influye en el estado de sus nodos padre. En sentido contrario, la modificación de un nodo padre no influye en sus hijos. Posteriormente se determina un nuevo punto mejoresNodos y los nodos correspondientes a la máximas diferencias se marcan como reforestadas en una matriz que almacena los resultados $(S=S \cup B)$. La Figura 8 muestra un ejemplo sencillo con una lista de nodos ordenados de acuerdo a la diferencia de flujo en la raíz (ld). Tomando en cuenta que el nodo \# 3 es el padre del node \# 2, el primero se remueve de las tres primeras posiciones de la lista. En consecuencia, los nodos \# 2 y \# 11 son seleccionados en esta iteración como los mejores a ser reforestados. La AE se actualiza en cada nodo (celda) marcada como reforestado y desde allí en todos los nodos en el sendero hasta llegar al nodo raíz (actualización de la $\mathrm{AE} \forall_{\text {node }} \in \mathrm{P}$ en el algoritmo 3). Una vez que los mejores nodos a ser reforestados son seleccionados luego de la primera llamada de LDR en el algoritmo 3, en las siguientes iteraciones estos nodos (conjunto S) harán uso de la función convexa lineal por segmentos para nodos reforestados para actualizar la AE y para determinar la cantidad de flujo de sedimentos que entregarán los nodos a sus padres.

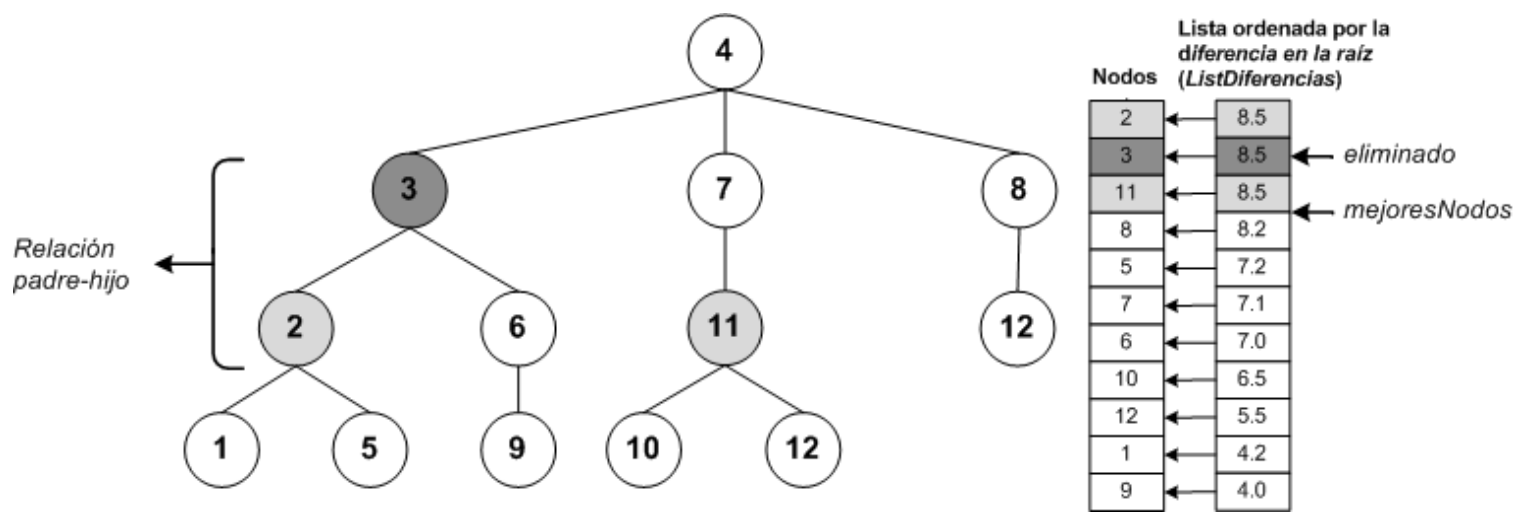

Figura 8. Localización de los mejores nodos.

El proceso para ubicar los mejores nodos (LBN, algoritmo 3) se itera hasta que un número determinado $(\varphi)$ de nodos (celdas) son seleccionados para ser reforestados (Mientras $|\mathrm{S}|<\varphi$ ).

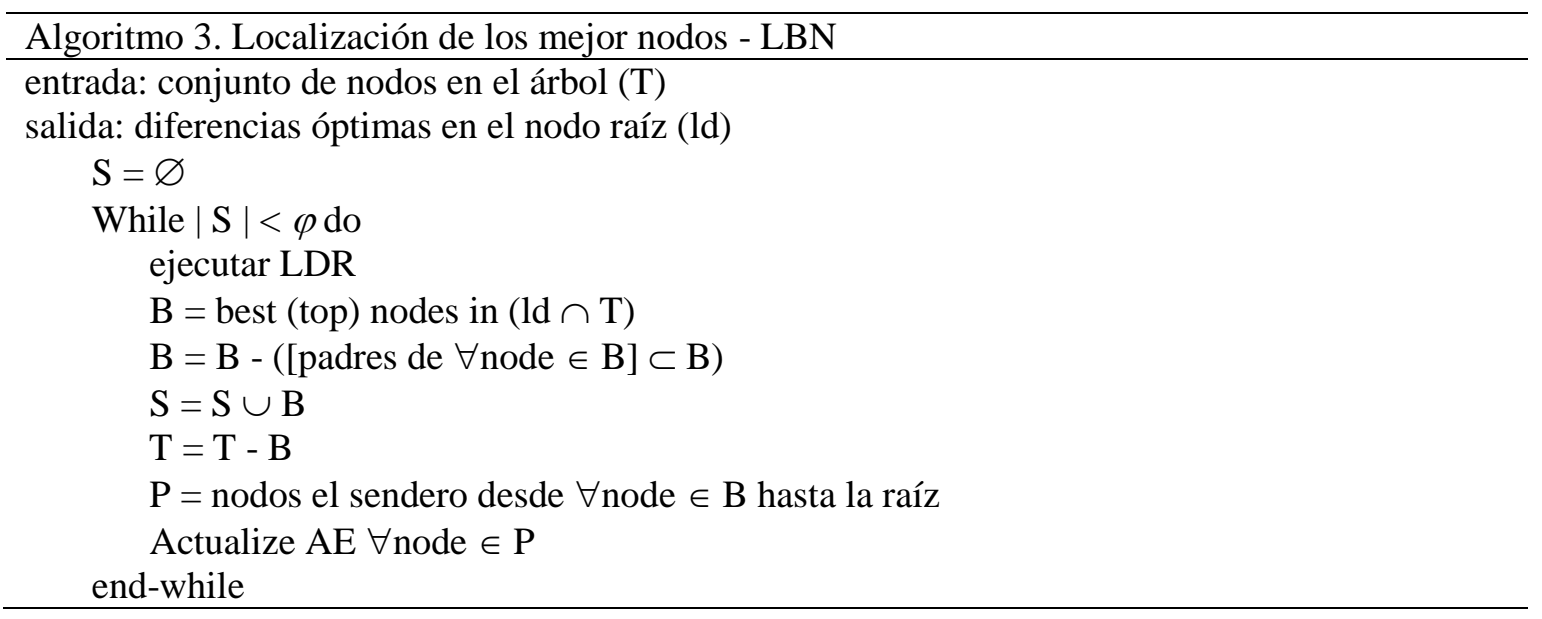

\section{RESULTADOS Y DISCUSIÓN}


Los datos se toman del conjunto de datos incluídos en el paquete de software para Windows Grass 6.3.0. Dos pequeñas cuencas en Dakota del Sur en los EEUU, formadas respectivamente por 536 (Cuenca 1) y 299 (Cuenca 2) celdas, se utilizan para probar ambos métodos para reducir al mínimo la carga de sedimentos en la salida (raíz) de una cuenca predeterminada. Los mapas raster en estas regiones están compuestos por celdas de $30 \mathrm{~m} \times 30 \mathrm{~m}$.

Los siguientes mapas se preparan en Grass 6.3.0 para cada prueba: dirección de flujo, pérdida de suelos por la erosión que representa la producción de flujo, un mapa con pendientes normalizadas para representar el factor de flujo y cauces de ríos. Mientras que valores 0,5 y 1,0 se especifican en cada celda de los mapas que representan los puntos de corte \# 1 y \# 2 respectivamente; nuevo punto de corte \# 1, nuevo punto de corte \# 2 y nuevo factor de flujo se especifican de acuerdo a los valores de prueba en el Tabla 1. Por último, la nueva producción de flujo $\left(\beta_{j}\right)$ mantiene los mismos valores que la producción inicial de flujo $\left(\alpha_{i}\right)$. Esta última condición no refleja condiciones reales, pero se utiliza debido a que los niveles de erosión después de la reforestación no están disponibles en el conjunto de datos. Sin embargo, esta condición no afecta la generalidad de los métodos. Las técnicas propuestas identifican un conjunto de celdas que permiten reducir al mínimo la carga de sedimentos en la salida de la cuenca hidrográfica cuando su función de flujo cambia, es decir, cuando el punto de corte \# 1, el punto de corte \# 2 y el factor de flujo se modifican. El Tabla 1 presenta los parámetros utilizados en las pruebas.

Tabla 1. Parámetros aplicados para probar las cuencas hidrográficas 1 y 2.

\begin{tabular}{lcclcrcc}
\hline Parámetro & \multicolumn{3}{c}{$\begin{array}{l}\text { Valores iniciales } \\
\text { celdas no reforestadas }\end{array}$} & \multicolumn{2}{c}{ Parámetro } & & \multicolumn{2}{c}{$\begin{array}{c}\text { Valores de prueba para } \\
\text { celdas reforestadas }\end{array}$} \\
\hline $\begin{array}{l}\text { Producción de } \\
\text { flujo }\end{array}$ & $\alpha_{j}$ & Erosión & $\begin{array}{l}\text { Nueva Prod. } \\
\text { flujo }\end{array}$ & $\beta_{j}$ & Erosión & Erosión & Erosión \\
\hline Factor de flujo & $\gamma_{j,}$ & Pendiente & $\begin{array}{l}\text { Nueva Fact. } \\
\text { flujo }\end{array}$ & $\delta_{j, k}$ & 0,2 & 0,3 & 0,2 \\
\hline Punto de corte 1 & $\sigma_{j,}$ & 0,5 & Nuevo P. corte 1 & $\sigma_{j, 1}$ & 1,0 & 1,0 & 0,5 \\
\hline Punto de corte 2 & $\sigma_{j,}$ & 1,0 & Nuevo P. corte 2 & $\sigma_{j, 2}$ & 2,0 & 2,5 & 1,0 \\
\hline
\end{tabular}

\subsection{Resultados numéricos y gráficos}

Mientras que el modelo lineal se implementa en Lingo v7, el método heurístico utiliza el lenguaje de programación Java. Ambos métodos son probados para reforestar números de celdas en el conjunto A $=(10,25,50,100,150,200)$. De acuerdo con la demostración en la sección 4, las pruebas con los dos métodos producen los mismos resultados en ambas cuencas. La Figura 9a muestra las condiciones iniciales para la cuenca hidrográfica 1: la producción de flujo (erosión), el factor de flujo (pendiente), y cauces de río. Los resultados para las seis cantidades de celdas en el conjunto A se muestran en la Figura 9b.

En la Figura $9 \mathrm{~b}$ se puede ver que las soluciones óptimas comprenden celdas con pendientes altas (factor de flujo). Esto sugiere que las celdas a ser seleccionadas como óptimas para ser reforestadas son los que facilitan el arrastre de sedimentos en las condiciones iniciales. Las celdas con alta erosión (producción de flujo) comienzan a aparecer cuando el número objetivo es de 100 celdas en adelante. Este comportamiento, que se encuentra también en las pruebas para el cuenca hidrográfica 2, está de acuerdo con la evaluación de expertos con respecto de erosión, pendientes y distancias a los lechos de los ríos. Sin embargo, la variación de los parámetros de la función de arrastre en las celdas reforestadas $\left(\sigma_{i, 3}, \sigma_{i, 4}, \delta_{i, j}\right)$, así como de la producción inicial $\left(\alpha_{i}\right)$ y nueva $\left(\beta_{i}\right)$ de flujo en cada celda puede dar lugar a un comportamiento diferente; por ejemplo, cuando las estrategias de reforestación especifican funciones de arrastre con capacidades de retención bajas, celdas con altos niveles de producción de flujo y ubicadas cerca de los lechos de río tendrán prioridad para ser reforestadas. 
a) Mapas iniciales

Erosión (Kfactor ${ }^{\star} 100 /$ Tfactor)/100 Pendiente (grados)
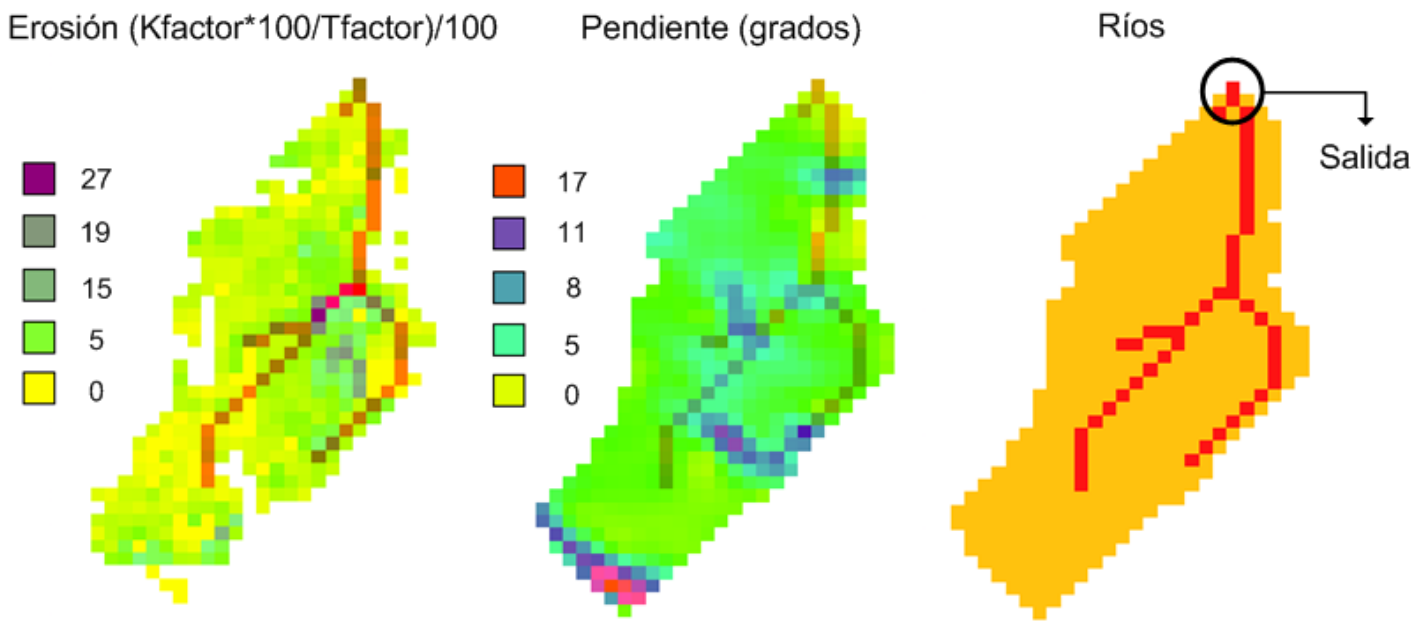

b) Mapas resultado

10 celdas óptimas

25 celdas óptimas

50 celdas óptimas
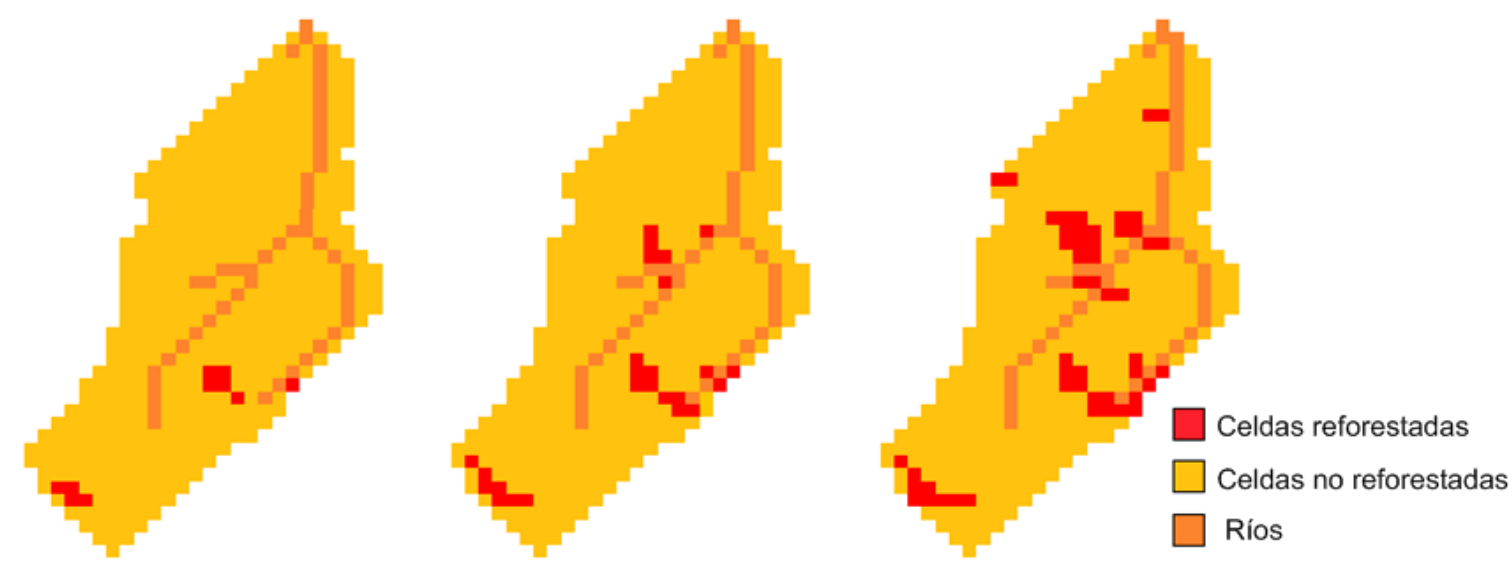

100 celdas óptimas

150 celdas óptimas

\section{0 celdas óptimas}
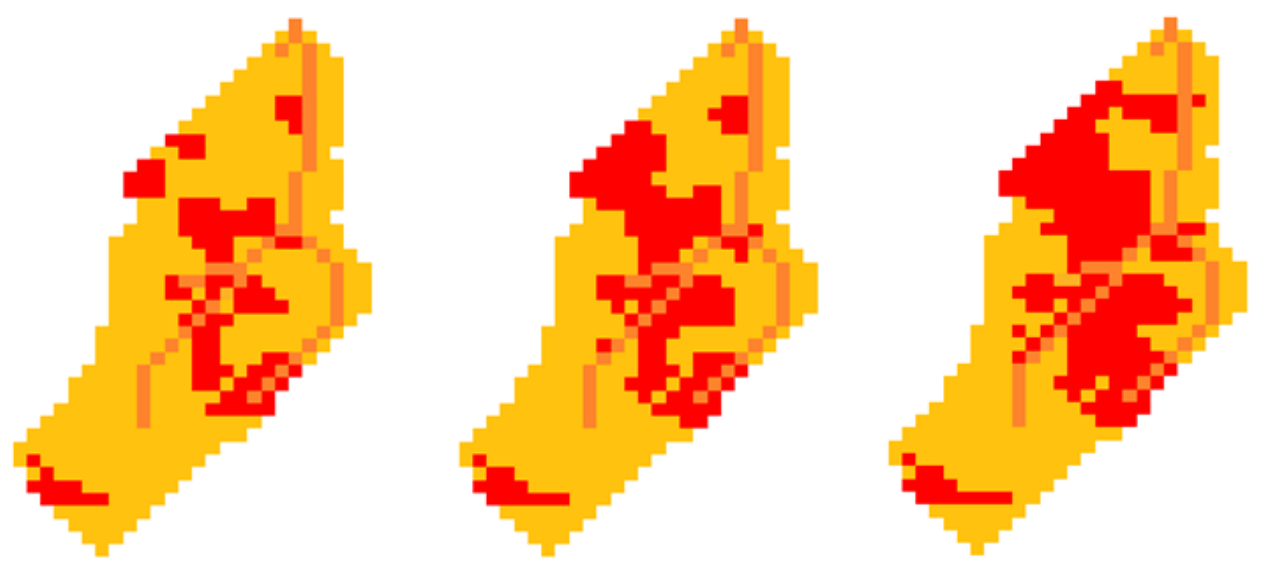

Figura 9. Resultados obtenidos al identificar celdas óptimas para reducir flujo en la salida de la cuenca \# 1 en Dakota del Sur.

Por otra parte, los valores objetivos aumentan en los casos de prueba 2 y 3 . Este comportamiento se produce debido a que el flujo de sedimentos llegará más fácilmente a la salida cuando el factor de 
flujo se incrementa de 0.2 a 0.3 en el caso de prueba 2 . En el mismo contexto, los segmentos más estrechos en la función de flujo explican los valores objetivo más alto para el caso de la prueba 3 (Tablas 1 y 2), es decir, menos flujo se retiene en cada nodo.

Tabla 2. Resultados de las pruebas en las cuenca hidrográfica 1 (tamaño $=536$ celdas).

\begin{tabular}{lcccccc}
\hline \multicolumn{1}{c}{ \# celdas } & 10 & 25 & 50 & 100 & 150 & 200 \\
\hline Valores de prueba 1 & & & & & & \\
Tiempo [segundos] & & & & & & \\
Heurística & 0,47 & 1,17 & 2,27 & 4,27 & 6,05 & 7,64 \\
F, Matemática & 113 & 48 & 46 & 46 & 46 & 46 \\
Valor objetivo & 1556,7 & 1541,1 & 1515,9 & 1467,4 & 1419,9 & 1373,3 \\
\hline Valores de prueba & & & & & \\
Tiempo [segundos] & & & & & \\
Heurística & 0,47 & 1,16 & 2,27 & 4,27 & 6,05 & 7,67 \\
F, Matemática & 2245 & 54 & 53 & 47 & 48 & 53 \\
Valor objetivo & 1557,7 & 1543,6 & 1520,9 & 1477,4 & 1434,9 & 1393,3 \\
\hline Valores de prueba 3 & \multicolumn{7}{c}{} & & & \\
Tiempo [segundos] & & & & & & \\
Heurística & 0,47 & 1,17 & 2,27 & 4,28 & 6,05 & 7,67 \\
F, Matemática & 47 & 54 & 47 & 76 & 48 & 47 \\
Valor objetivo & 1565,7 & 1563,5 & 1560,7 & 1557,1 & 1554,5 & 1552,8 \\
\hline
\end{tabular}

Tabla 3. Resultados de las pruebas en las cuenca hidrográfica 2 (tamaño $=299$ celdas).

\begin{tabular}{lcccccc}
\hline \multicolumn{1}{c}{ \# celdas } & 10 & 25 & 50 & 100 & 150 & 200 \\
\hline $\begin{array}{l}\text { Valores de prueba 1 } \\
\text { Tiempo [segundos] }\end{array}$ & & & & & & \\
Heurística & 0,19 & 0,45 & 0,89 & 1,58 & 2,14 & 2,58 \\
F, Matemática & 12 & 13 & 12 & 13 & 17 & 13 \\
Valor objetivo & 770,3 & 753,6 & 726,8 & 675,9 & 628,8 & 608,9 \\
\hline Valores de prueba & & & & & & \\
Tiempo [segundos] & & & & & & \\
Heurística & 0,19 & 0,45 & 0,86 & 1,58 & 2,14 & 2,58 \\
F, Matemática & 11 & 11 & 11 & 11 & 11 & 11 \\
Valor objetivo & 771,2 & 756,1 & 731,7 & 685,8 & 643,6 & 624,6 \\
\hline Valores de prueba 3 & & & & & & \\
Tiempo [segundos] & & & & & & \\
Heurística & 0,19 & 0,47 & 0,88 & 1,58 & 2,19 & 2,63 \\
F, Matemática & 11 & 11 & 11 & 12 & 17 & 13 \\
Valor objetivo & 779,2 & 775,9 & 771,6 & 765,2 & 762,1 & 761,4 \\
\hline
\end{tabular}

En los Tablas 2 y 3, el tiempo de cómputo requerido por el método heurístico se incrementa linealmente con el número de celdas reforestadas y estos tiempos en ningún caso son mayores que el menor tiempo registrado por la formulación matemática. Por otra parte, los tiempos requeridos por el enfoque matemático no siguen una clara relación con el número de celdas reforestadas (por ejemplo, el caso de prueba 2 para identificar 10 celdas en la cuenca 1), muy probablemente estos tiempos dependen de los datos particulares en los mapas raster, lo que determina el número de soluciones óptimas o cerca de lo óptimo a analizar.

\section{CONCLUSIONES}


El presente trabajo ha desarrollado una formulación entera (IP) y un método heurístico (CAMF) para la localizar celdas que permitan reducir al mínimo la carga de sedimentos a la salida de una cuenca hidrográfica. Los resultados de la formulación entera son tomados como una referencia óptima para medir la precisión del método heurístico.

Bajo las condiciones de los casos de prueba, los resultados de la formulación matemática y de CAMF reflejan que celdas con una pendiente alta tienen prioridad para ser incluidas en la solución final. Adicional a la pendiente (factor de flujo), la distancia al lecho de los ríos y la producción de flujo (erosión) también determinan la selección de celdas. Este comportamiento puede variar con los parámetros especificados en la función de arrastre de flujo para las celdas seleccionadas $\left(\sigma_{i, 3}, \sigma_{i, 4}, \delta_{i, j}\right)$, así como con la producción inicial $\left(\alpha_{j}\right)$ y nueva $\left(\beta_{j}\right)$ de flujo en cada celda.

Los tiempos de computo (Tablas 2 y 3 ) empleados por la formulación matemática permiten concluir que el modelo CAMF tiene un rendimiento superior para la localización de celdas que reduzcen al mínimo la carga de sedimentos a la salida de una cuenca hidrográfica. Estos resultados, y considerando la inmensa cantidad de memoria necesaria para almacenar las variables de la formulación matemática, permiten concluir que el modelo CAMF es apropiado para aplicarlo en problemas de tamaño real a nivel regional.

\section{BIBLIOGRAFÍA}

Ahuja, R.K., T.L. Magnanti, J.B. Orlin, 1993. Network Flows: Theory algorithms, and applications. Prentice-Hall.

Bogaart, P.W., P. Torfs, A. Troch, 2006. A multiple flow direction algorithm for computing intra hillslope length maps. Geogr. Res. Abstr., 8.

Coulthard, T.J., M.J. Kirkby, M.G. Macklin, 1999. Fluvial Process and Environmental Change, chapter Modelling the impacts of Holocene environmental change in an upland river catchment, using a cellular automata approach, John Wiley and Sons Ltd., 31-46.

Coulthard, T.J., M.G. Macklin, M.J. Kirkby, 2002. A cellular model of holocene upland river basin and alluvial fan evolution. Earth Surf. Processes, 27, 269-288.

D'Ambrosio, D., S. Di-Gregorio, S. Gabriele, and R. Gaudio, 2001. A cellular automata for soil erosion by water. Phys. Chem. Earth, 26, 33-39.

Desmet, P.J.J., G. Govers, 1996. Comparison of routing algorithms for digital elevation models and their implications for predicting ephermal gullies. Int J Geogr Inform Syst, 3,311-331.

Di-Gregorio, S., R. Serra, 1999. An empirical method for modelling and simulation some complex macroscopic phenomena by cellular automata. Future Gener. Comp. Sy., 657, 1-13.

Failfield, J., P. Leymarie, 1991. Drainage networks from grid digital elevation models. Water Resour. Res., 27, 709-717.

Longley, P.A., M.F. Goodchild, D.J. Maguirre, 2005. Geographic Information Systems and Science, Second Edition. John Wiley and Sons Ltd.

Murray, A.B., C. Paola, 1994. A cellular model of braided rivers. Nature, 371, 54-57.

Parsons, J.A., M.A Fonstad, 2007. A cellular automata model of surface water flow. Hydrol. Process., 21, 2189-2195.

Qin, C., A.X. Xhu, T. Pei, B. Li, C. Zhou, L. Yang, 2007. An adaptative approach to selecting a flowpartition exponent for a multiple-flow-direction algorithm. Int. J. Geogr. Inf. Sci., 21, 443-458.

Van Orshoven, J., 2007. Introduction to spatial data modelling and functionality of geospatial technology. Department of Earth and Environmental Sciences, K.U.Leuven, Leuven, Belgium.

White, R., G. Engelen, 2000. High-resolution integrated modelling of the spatial dynamics of urban and regional systems. Environ. Urban Syst. Comput., 24, 383-400.

Winston, W.L., 1994. Operations Research, Applications and Algorithms. Int. Thomson Publ.. 
MASKANA, Vol. 1, No. 1, 2010

Wolock, D.M., G.J. McCabe Jr., 1995. Comparison of single and multiple flow direction algorithms for computing topographic parameters in TOPMODEL. Water Resourc. Res., 31, 1315-1324. 